\title{
JOAN BARTER, TRAYECTORIA DEL GRAN ORGANISTA Y RELIGIOSO DEL BARROCO ESPAÑOL*
}

\author{
JOAN BARTER, CAREER OF THE GREAT SPANISH ORGANIST AND \\ RELIGIOUS OF THE BAROQUE PERIOD
}

\author{
SANDRA SOLER CAMPO \\ Universidad de Barcelona
}

Recibido: 13/03/2020 Aceptado: 24/06/2020

\section{RESUMEN}

Joan Barter nació en Mequinenza (Zaragoza) en una fecha no precisada del año 1648. Fue compositor de música instrumental y vocal religiosa, tanto en latín como en lengua castellana. Su obra conservada, aunque no muy extensa, nos muestra, a un compositor de gran calidad y dominio del contrapunto, al que puede considerarse a través del estudio de sus obras artísticas como uno de los grandes autores del barroco español.

Se dispone de pocos datos sobre su familia. Siendo aun niño, ingresó en la escolanía de la Catedral de Lleida, y allí, al tiempo que ejercía de cantor, se formó musicalmente con el maestro de capilla Juan Merino. A partir de un testamento, datado el 9 de octubre de 1710, del mequinenzano Pere Soro Barter “domer" (administrador) de la Catedral de

${ }^{*}$ Un profundo y sentido agradecimiento al historiador mequinenzano Jordi Estruga, quien ha cedido su trabajo de investigación en torno a la vida y obra del músico Joan Barter y quien nos ha dejado recientemente. 
Barcelona, se conoce que el prevere de la misma catedral, Joan Barter, era tío suyo. Los dos, tío y sobrino, fueron unos reconocidos mequinenzanos de finales del siglo XVII e inicios del siglo XVIII, con cargos de responsabilidad en la Catedral de Barcelona. Barter falleció el 5 de Diciembre del año 1706. Sus restos reposan en el claustro de la Catedral de Barcelona, al lado de su sobrino Pere Soro Barter.

En el presente artículo, se hará un recorrido biográfico en el que se mencionarán las principales obras musicales escritas por este compositor poco desconocido por nuestra sociedad.

Palabras clave: maestro de capilla, compositor, música, religioso, contrapunto, villancico, catedral.

\section{ABSTRACT}

Joan Barter was born in Mequinenza (Zaragoza) in an unknown date of the year 1648. He was a composer of instrumental and religious vocal music, both in Latin and Spanish. His preserved work, although not very extensive, shows us, a composer of great quality and mastery of counterpoint, which can be considered through the study of his artistic works as one of the great authors of the Spanish Baroque.

Few data are available about his family. When he was a child, he joined the choir at Lleida Cathedral, and there, while working as a singer, he received his musical training from the chapel master Juan Merino (Scala Aretina, 2001). From a will, dated 9 October 1710, of the Mequinenzano Pere Soro Barter "domer" (administrator) of the Cathedral of Barcelona, we know that the prevere Joan Barter was his uncle. They were prestigious people from Mequinenza between the end of the 17th century and the beginning of the 18th century, with positions of responsibility in the Cathedral of Barcelona. Barter died on 5th December 1706. His rest remains in the cloister of the Cathedral of Barcelona, next to his nephew Pere Soro Barter.

In this article, there will be a biographical sketch in which the main musical works written by this little known composer by our society will be mentioned.

Keywords: chapel master, composer, music, religious, counterpoint, villancico, cathedral 


\section{TRAYECTORIA DE JOAN BARTER: VIDA RELIGIOSA Y MUSICAL}

Desde el año 1661 Joan Barter fue niño cantor en la escolanía de la Catedral de Lérida, formándose junto al maestro Joan Merino. Durante el paso de la niñez a la etapa adulta experimentó la muda de la voz, pero no abandonó la música, destacando como organista. A principios del año 1664 Barter recibió una gratificación económica del Capitolio de la Sede de Lérida por los servicios prestados a la capilla hasta esa fecha. Continuó entonces como ayudante del maestro Merino hasta que éste ganó el año 1668 el magisterio en la catedral de Orense (Salisi, 1998). Joan Barter fue nombrado maestro interino de la Sede de Lérida, cargo que fue confirmado sin oposición el 7 de septiembre de 1668.

Entre su producción musical conservada encontramos fundamentalmente música religiosa: misas, salmos, un magnificat y diversos villancicos de temática religiosa. Alguno de los villancicos es considerado como tono debido a su carácter intimista y por estar escrito en romance. Desde 1668 hasta 1682 Barter compuso "Misa a seis voces y bajo continuo". En 1670 escribió "Letras de los villancicos" lo cuales se cantaron en la noche de Navidad en la Catedral de Lérida (Torrente y Marín, 2000). Un año después, en 1671 se imprimió en Barcelona la publicación de Raphael Figueró "Villancicos" interpretándose la noche de Navidad en la Santa Iglesia de Lérida", siendo Maestro de Capilla el Racionero Joan Barter.

En Febrero de 1682 falleció el maestro de capilla de la Catedral de Barcelona Lluís Vicenç Gargallo. Posteriormente fueron convocadas las oposiciones para cubrir la plaza que había quedado vacante y que fue ganada por Joan Barter. El 13 de Julio del 1682 Joan Barter deja la Catedral de Lérida al obtener la plaza y se traslada a la ciudad condal. El mismo día, se da la licencia a Joan Barter para ir a Lérida y poder así despedirse de la Sede y organizar sus pertenencias. Probablemente ya fue en Barcelona cuando compuso el "El Magnificat a 8 voces y bajo continuo", considerada una composición altamente original y atípica en la música barroca española (Scala Aretina, 2002).

Barter potenció la labor docente en la escolanía de la catedral de Barcelona, llegando a ceder una sala de sus dependencias como sala de estudio de la escolanía. Así mismo, intentó mejorar la calidad y cantidad de los efectivos de la capilla de música contratando nuevos cantores.

El 8 de diciembre de 1685 se publica su obra titulada "Villancicos" la cual se interpretó en la Santa Catedral Iglesia de Barcelona en el Reverendo, Obsequioso y Sacro Culto y su Octavario, dedicado a la Purísima e Inmaculada Concepción de N.S. 
En 1686 Barter intervino de jurado en las oposiciones al magisterio de la sede de Lérida y posteriormente en 1688 en Girona. El 7 de Diciembre de 1696, Barter solicitó la jubilación y fue sustituido como maestro de capilla por su discípulo, el célebre Francesc Valls ${ }^{1}$, maestro de la capilla de Santa María del Mar de Barcelona.

Durante el periodo comprendido entre los años 1688 al 1696, Joan Barter realiza diversos conciertos de villancicos. Compone además música para la fiesta de la Cofradía de la Purísima e Inmaculada Concepción, fundada en el Real Palacio de los Invictissimos Señores Reyes de Aragón, Condes de Barcelona, y posteriormente transferida a dicha Santa Iglesia. Ofreció un concierto de 3 villancicos en 1688 (Codina, 2003). Dos año más tarde, lo hizo en la Catedral de Barcelona, en el Convento de San Josef y en la Orden de Carmelitas. En 1691 para una fiesta de acción de gracias promovida por los mayorales del San Cristo de la Ribera de San Juan ofrece un concierto de 3 villancicos (Codina, 2003)
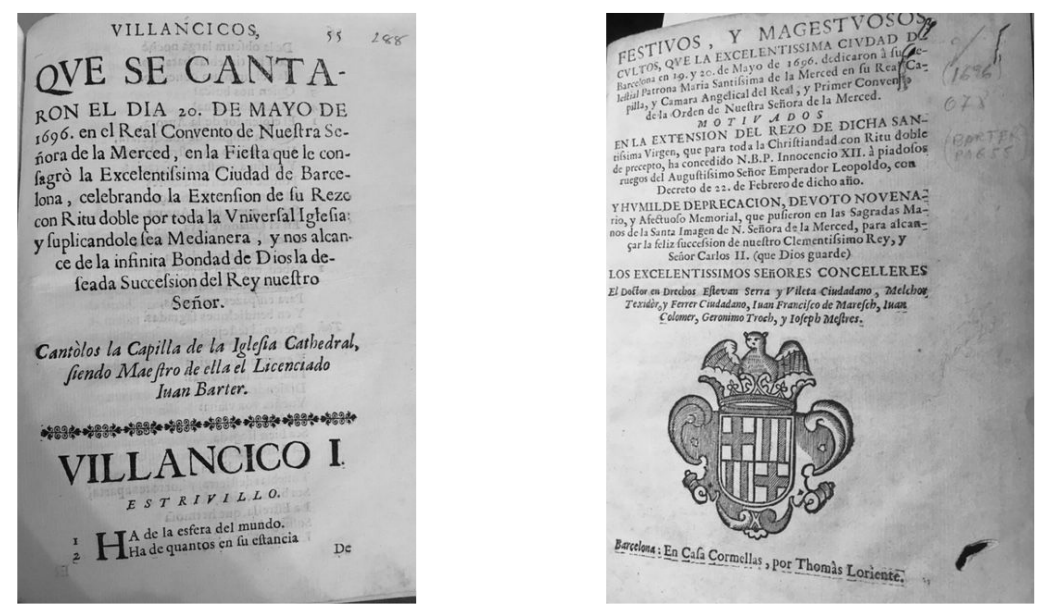

Imagen 1 y 2: Página 55 y portada de la publicación de Thomas Loriente, Barcelona 1696, con villancicos de Joan Barter dedicados a María Santísima de la Merced (Biblioteca de Jordi Estruga).

1 El Coro de Cámara Francesc Valls, adoptó el nombre en honor al maestro de capilla de la Catedral Francesc Valls Galán (Barcelona?, ca. 1671 -1747). Valls fue uno de los máximos exponentes de la música barroca en Cataluña. Fue discípulo de Miquel Rosquelles. Fue maestro de capilla de la iglesia parroquial de Mataró y, en 1688, de la catedral de Girona. Posteriormente, fue nombrado maestro de capilla de Santa María del Mar el año 1696 y, el 22 de diciembre del mismo año, sustituyó a Joan Barter, que se retiraba, en la Catedral de Barcelona. El 18 de febrero de 1706, tras la muerte de Barter, consiguió su propio nombramiento perpetuo. Se jubiló en 1726, aunque siguió siendo propietario de la plaza hasta su muerte, cuando fue sustituido por Joan Galvany Nonell. 
En su nuevo destino como maestro de capilla de la Catedral de Barcelona, Joan Barter, se implicó totalmente en la formación de la escuela coral de la Catedral e introdujo nuevos instrumentos musicales tales como violines y oboes. Sus obras tuvieron un éxito considerable y fueron interpretadas en diferentes Iglesias de Cataluña. Gracias a ello se han conservado algunas de sus partituras que han podido llegarnos hoy en día. Un ejemplo de ello es la "Lamentación a 8 voces" de 1687 encontrada en la Parroquia de la Iglesia de Verdú en Urgell, Lérida.

Joan Barter es considerado como un compositor cuya personalidad musical estaba muy definida y era a la vez original. Así, buscaba una nueva expresividad mediante el uso de instrumentos solistas, las variaciones rítmicas y de tempo. Fue un músico que desarrolló su obra durante el periodo de transición entre el "stile antico" y el "stile moderno".

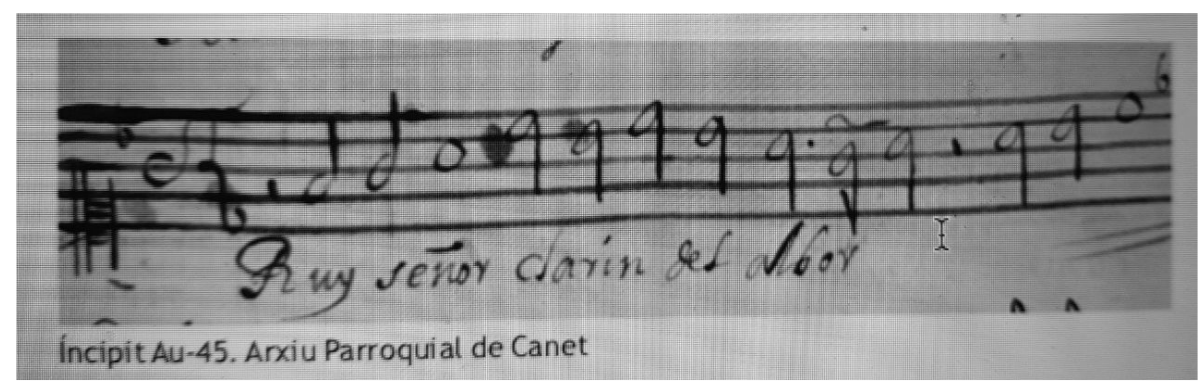

Imagen 3: Ruyseñor de Joan Barter. «Barter / Tono solo a la Pura Concepción / Ruyseñor clarín del albor». Íncip literario: Ruyseñor clarín del albor. Voz solista: Instrumentos, Violon, Acompañado de arpa Tonalidad: Fa Mayor.

Formato: 3 folios apaisados.

Este tono ${ }^{3}$ forma parte de la treintena de obras escritas en romance que se han conservado de Joan Barter, en las cuales el autor se muestra heredero de la tradición estilística y expresiva iniciada por sus antecesores religiosos y a la vez

2 El stilo antico y el stilo moderno son dos prácticas interpretativas y de composición vocal e instrumental. El stilo antico generalmente indica la presencia de rasgos asociados al Renacimiento y a la figura del compositor Palestrina. Después de su muerte en 1594 comienzan a surgir prácticas compositivas diferentes, y aquellos autores que siguieron componiendo al estilo de Palestrina (stile antico) se consideraron autores cuyas composiciones eran poco innovadoras.

3 Los tonos son melodías (música vocal) escritas en romance durante el siglo XVII. El término tono, se tomó prestado del Cancionero Musical y Poético del siglo XVII el cual fue recopilado por Claudio de la Sablonara. Este también se conoce como Cancionero de Munich o Cancionero de la Sablonara. 
maestros de capilla Joan Cererols, Vicente Gargallo y Francesc Soler. En este tono Barter articula el discurso compositivo a través del diálogo entre la voz y el violín solista siguiendo un dinamismo imitativo propiamente de estilo barroco.

La vida musical religiosa del 1600 en Barcelona es en general poco conocida. El siglo XVII fue un siglo de convulsiones, revueltas y guerras, de hambre y de epidemias aunque una de las cosas que caracterizó este periodo de cien años fueron la transformación y la evolución artística. En el ámbito musical, ello se percibe en la coexistencia de los mencionados stilo antico y stilo moderno y en el solapamiento de los últimos alientos del Renacimiento y del Manierismo con la primera etapa del estilo barroco. En Cataluña, la catedral de Barcelona fue, junto con el Monasterio de Montserrat, el principal centro de la producción musical "de autor" en Cataluña.

Las composiciones de Joan Barter se interpretaron en un considerable número de catedrales e iglesias del país. Sus obras pueden encontrarse en diferentes iglesias de Cataluña, sobretodo en el archivo comarcal de la Segarra, en Cervera y en la Biblioteca de Catalunya, en Barcelona, formando parte del "Fons musical de la parroquia de Santa María de Verdú”.

Cuatro fueron los maestros de capilla más importantes que ejercieron su cargo a lo largo de esos cien años en Barcelona, y que a la vez nos ilustran cuatro etapas básicas del controvertido y mal conocido como barroco seiscentista. Joan Barter fue uno de ellos, testigo de cómo nuevos instrumentos más modernos, como violines y oboes, iban integrándose en la capilla de música de la catedral ${ }^{4}$, representando el paso hacia el Barroco más "sinfónico" del siglo XVIII. Del total de las 489 obras de este periodo, 370 pertenecen a 66 autores y el resto son anónimas. Entre las diferentes obras se encuentran villancicos escritos en lengua catalana. Observemos la distribución de las obras: escritas en catalán: 8 , en castellano: 126, en latín: 343, (además de los 164 fragmentos de canto llano), en castellano y latín: 3 , sin texto: 9 .

Algunas de estas eran de Joan Barter: "Querubes y hombres", villancico a doce voces, "Cantaré, virgen pura," villancico a cinco voces, "Al amor que galán

4 Las capillas de música fueron creadas para solemnizar los diversos actos que se llevaron a cabo en las iglesias. Además se usaron como instrumento de magnificencia de celebraciones religiosas, que servía también para amenizarlas. A la vez era un hecho propagandístico. A finales del siglo XVI, fue cuando se empezaron a consolidar las capillas pero la época más esplendorosa de las musicales fue durante los siglos XVII y XVIII. La figura más importante de la capilla era su maestro. Este era el máximo responsable de su organización, incluyendo los ministriles o instrumentistas. También era el encargado de componer de las obras que debían interpretarse durante las celebraciones, y era también el responsable de la educación musical de los niños cantores de la capilla. 
hermosea", villancico a diez voces, "Sosiegos del ocio" tono, cuatro voces y bajo continuo. Una de sus composiciones más complejas es la "Missa a dos triples", denominada así por el papel solista que realiza el dueto de sopranos el cual actúa como primer coro y posteriormente dialoga con el segundo. Esta misa la conforman las cuatro voces: soprano, contralto, tenor y bajo. Está compuesta íntegramente en el primer tono gregoriano aunque con la transposición queda convertida en una obra que gira siempre alrededor de la tonalidad de Sol menor.

Los recursos expresivos barrocos utilizados en ella son fundamentalmente: líneas melódicas que descienden al cantarse las palabras "et descendit de cælis" o "et sepultus est", líneas ascendentes en "et resurrexit" y "et ascendit in cælum", o figuraciones rítmicas percutidas en "Dominus Deus sabatho". Como en un lenguaje concertante, el segundo coro interactúa con el primero en combinación equilibrada, pero a menudo glosa o complementa las intervenciones del mismo en un modo casi "orquestal".

Otra de sus obras a destacar (cuya letra puede leerse a continuación) es "Sosiegos del ocio indigno” de Joan Barter, (año 1677) texto de autor anónimo.
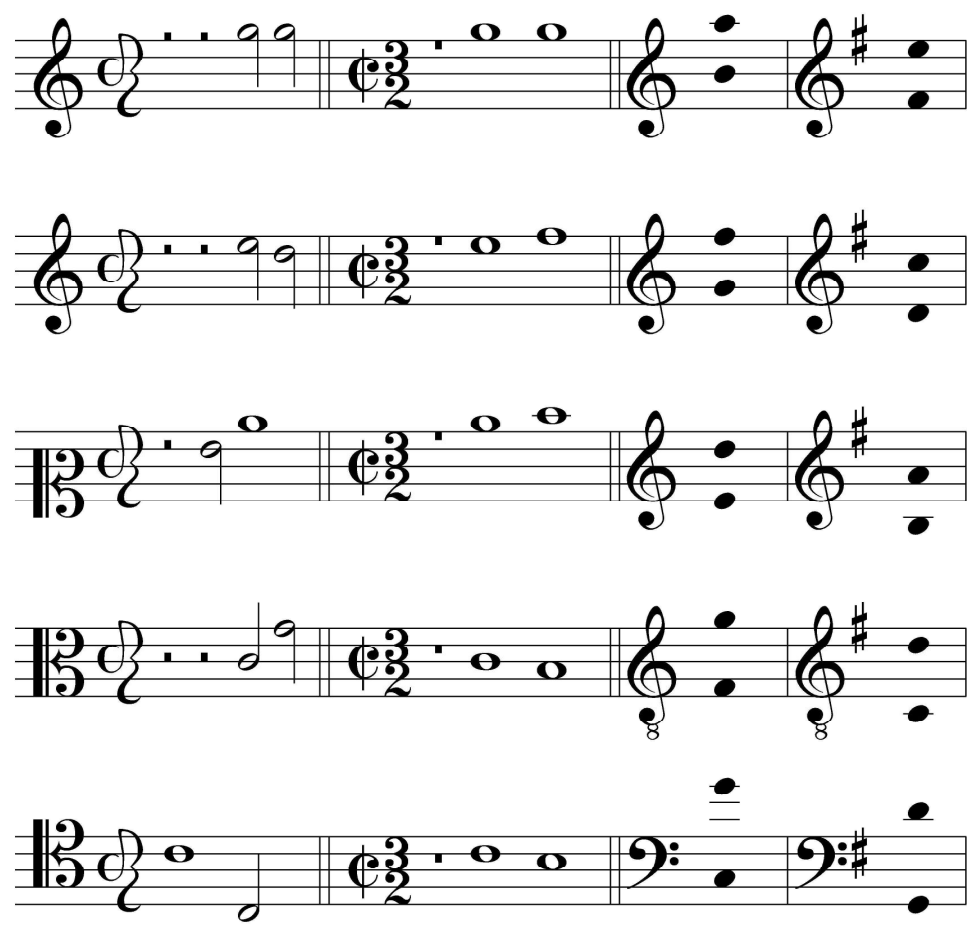

Imagen 4: Barcelona. BC, M. 1677-/23, “Tono a 4, al humano. Sosiegos del ocio. J. Barter". 


\section{Letra}

Sosiegos del ocio indigno más que felicidades son delitos.

[1a]

Pensamiento, que, aspirando

al noble afán de rendido, 5

construyes del vasallaje blasones a tu albedrío,

[2a]

procura esmerar, constante

(con atenciones de fino), rendimientos que coronen 10 aciertos de lo electivo.

[3a]

Anhela a formar el culto, pero explícale remiso que pueden culpar finezas liviandades de atrevido. 15

[4a]

No que tu amor insinúen consientas a tus sentidos, que nunca será más grande que cuando más recogido.

[5a]

Pausa de tus penas todas 20 llegas a hacerte a ti mismo, pues labras del alto empleo, en tus ideas, tu alivio.

\section{PRESENCIA DE LAS OBRAS MUSICALES DE JOAN BARTER EN LA} ACTUALIDAD

En Enero de 1991, se registra en los Estudios Albert Moraleda de Barcelona, el CD que publicó "la mano de guido" DIATESASARON de la obra de Joan Barter, "Trio-sonata en fa mayor" Allegro- Andante- Allegro (partitura transcrita por Lothar Siemens).

En 1998, se cita una relación de 22 obras de Joan Barter en el artículo "Las partituras de la capilla de música de Santa María de Verdú", siglos XI-XVIII 
(Salisi, 1998). El 20 de Diciembre del mismo año, en el artículo de Ricard García Moya, titulado "El villancico de las naciones y los valencianos", hace referencia al villancico del año 1671 de Joan Barter.

El 2 de Julio del año 2000, nace una formación con el objetivo de recuperar la música de la Catedral de Girona. Un grupo de jóvenes instrumentistas, musicólogos, y cantores, recuperan piezas inéditas que presentaran al final de la misa de once en la Catedral de la citada ciudad, interpretando composiciones de Francesc Soler y Joan Barter.

En 2001 Scala Aretina Ediciones Musicales, dentro de la serie A: música vocal en latín, volumen $3 \mathrm{~A}$, publica "Misa a 6 voces y Bajo continuo" de Joan Barter. El año siguiente Scala Aretina Ediciones Musicales, dentro de la Serie A: música vocal en latín, volumen 11 A, publica "Magnificat de 8 voces y Bajo Continuo" de Joan Barter. Se trata de una composición original y atípica dentro de la música barroca española. Por el momento parece tratarse de un ejemplo único dentro del ámbito hispánico (Archivo capitular de la Catedral de Barcelona).

En 2003, dentro de la colección Scripta et Documenta 64, la Abadía de Montserrat publicó "Catalogo de Villancicos y oratorios impresos de la biblioteca de Montserrat" siglos XVII-XIX. Su autor Daniel Codina i Giol, hace referencia a las obras de Joan Barter (Archivo capitular de la Catedral de Barcelona).

El 15 de Noviembre del año 2009, la formación Scala Aretina, dio un concierto Barroco en catalán, un domingo 15 de Noviembre en la Ermita de Sant Pau. 400 personas asisten a los conciertos "más que música". En Sant Pere de Ribes, la formación Scala Aretina ofrece un concierto del Barroco musical catalán, el espectáculo incluía una pieza de Joan Barter.

El 2 de Julio del año 2010, un grupo de cantantes, instrumentistas y musicólogos, constituyeron Música antigua de Girona, interpretando con instrumentos de la época, la música que escribieron e interpretaron los músicos de la capilla de la Catedral de Girona, durante los siglos XVII y XVIII. Se llevó a cabo una presentación de esta iniciativa el 2 de Julio en la Catedral de Girona. Entre las piezas escogidas interpretaron una de Joan Barter muy vinculada a Girona, titulada "Respirad flores fragancias".

El 22 de Julio del mismo año 2010, l'Ajuntament de Terrassa organiza dentro del marco del "Barroco catalán" la música íntima en el programa "El ciclo del amor divino" se interpretó en la Iglesia del Convento de Sant Francesc la obra de Joan Barter, "Ruyseñor, clarín del albor". 
En 2012 se publica un volumen de Música en silencio del autor Lluís Marc Herrera, donde se rescata del olvido, en forma de extenso diccionario, las bibliografías de personajes que en su día protagonizaron la vida musical de las tierras de Lleida y del Pirineo. Recoge un centenar de músicos, intérpretes, compositores, musicólogos, directores, profesores de música, organistas y maestros de capilla de Lleida y de su territorio al largo de los siglos XVIII, XIX, y las primeras décadas del siglo XX.

En 2013 se exhibe la partitura en la Casa Museo del Ayuntamiento de Canet de Mar, dicha partitura forma parte de la treintena de obras escritas en romance que se han conservado de Joan Barter, con la intención de difundir el fondo de la capilla de música del archivo parroquial. En ellas el autor se muestra heredero de la tradición estilística y expresiva iniciada por sus antecesores en el género.

En el año 2013 el Consejo Superior de Investigaciones Científicas, publica: "Todo es amor. Manojuelo poético musical de Barcelona". Una antología de tonos humanos, teatrales y divinos conservados en la misma biblioteca donde figura Joan Barter.

Entre el 10 de Febrero y el 8 de Marzo del año 2014, se menciona la obra de Joan Barter, "Misa Scala Aretina" en la exposición de la Biblioteca de Catalunya, la obra "Tono Al Solo Con Xirimía y a Solo...".

El 16 de noviembre de 2014, los maestros de capilla de la Catedral de Barcelona, del siglo XVII con el coro de cámara de Francesc Valls, interpretan un programa dedicado a los maestros del siglo XVII. En la primera parte se interpretó la misa a 8 voces y bajo continuo de Lluís Vicenç Gargallo, el "Magnificat del primer tono", a 8 voces, y bajo continuo de Joan Barter y la "Salve Regina" de Miquel Selma. En la segunda, se interpretó la misa del cuarto tono a 6 voces y bajo continuo de Francesc Valls, y el cantante Domino de Joan Pau Pujol.

\section{CONCLUSIÓN}

Con todas estas obras se abrazan 100 años de la música que se escuchaba en Cataluña desde el renacimiento tardío al barroco. La música de Barter y de sus contemporáneos todavía no se caracterizó por la libertad creativa hasta la llegada y posterior eclosión melódica del barroco pleno.

La vida y obra de muchos autores del renacimiento o épocas posteriores tales como Monteverdi, Johan Sebastian Bach, Vivaldi entre otros podemos escucharla en los principales teatros, lugares de culto o salas de concierto de la mayoría de capitales europeas. Sin embargo no ocurre lo mismo con la música 
de Joan Barter. A pesar de que en su momento gozó de cierto reconocimiento tanto a nivel religioso como artístico, se deberían programar y continuar interpretando las creaciones musicales de Barter, gran músico del barroco Español tan poco conocido por nuestra sociedad.

\section{REFERENCIAS BIBLIOGRÁFICAS}

Barter, Joan. Misa a 6 voces y bajo continuo. Mollerussa: Scala Aretina, 2001. Barter, Joan. Magnificat a 8 voces y bajo continuo. Mollerussa: Scala Aretina, 2002.

Codina, Daniel. Catàleg dels villancicos $i$ oratoris impresos de la biblioteca de Montserrat. Segles XVII-XIX. Barcelona: Publicacions de l'Abadia de Montserrat, 2003.

Salisi, Josep María. "Las partituras de la capilla de música de Santa María de Verdú. Siglos XI-XVIII”. URTX revista cultural del Urgel núm 11 (1998): 69-88.

Torrente, Álvaro y Marín, Miguel Ángel. Pliegos de villancicos en la British Library (Londres) y la University Library (Cambridge). Kassel: Edition Reichemberger, 2000.

Sandra Soler Campo

Universidad de Barcelona

Facultad de Educación Passeig de la Vall d'Hebron, 171 08035 Barcelona (España) https://orcid.org/0000-0002-5560-1415 